\title{
Statistical Properties of Gamma-Ray Burst Host Galaxies $\dagger$
}

\author{
J. M. Chen ${ }^{1}$, L. W. Jia ${ }^{2}$ and E. W. Liang*1 \\ ${ }^{* 1}$ Department of Physics and GXU-NAOC Center for Astrophysics and Space Sciences, \\ Guangxi University, Nanning 530004, China; \\ email: lew@gxu.edu.cn \\ ${ }^{2}$ College of Physics and Electronic Engineering, Guangzhou University, Guangzhou 510006, \\ China.
}

\begin{abstract}
GRBs are the most luminous events in the Universe. They are detectable from local to high- $z$ universe and may serve as probes for high- $z$ galaxies (e.g., Savaglio et al. 2009; Kewley \& Dopita 2002). We compile the observations for 61 GRB host galaxies from literature. Their redshifts range from 0.0085 to 6.295 . We present the statistical properties of the GRB host galaxies, including the stellar mass $\left(M^{*}\right)$, star-forming rate (SFR), metallicity $(Z)$, extinction $\left(A_{V}\right)$, and neutral hydrogen column density $\left(N_{\mathrm{H}}\right)$. We explore possible correlations among the properties of gamma-ray burst host galaxies and their cosmic evolution with observations of 61 GRB host galaxies. Our results are shown in Figure 1. A clear $Z-M^{*}$ relation is found in our sample, which is $Z \sim M^{0.4}$. The host galaxies of local GRBs with detection of accompanied supernovae also share the same relation with high- $z$ GRB host galaxies. A trend that a more massive host galaxy tends to have a higher star-formation rate is found. The best linear fit gives a tentative relation, i.e, $S F R \sim M^{0.75}$. No any correlation is found between $A_{V}$ and $N_{H}$. A GRB host galaxy at a higher redshift also tends to have a higher $S F R$. Even in the same redshift, the $S F R$ may vary over three orders of magnitude. The metallicity of the GRB host galaxies is statistically higher than that of the QSO DLAs. The full version of our results please refer to Chen et al. (2012).
\end{abstract}

Keywords. gamma rays: bursts — galaxies: evolution
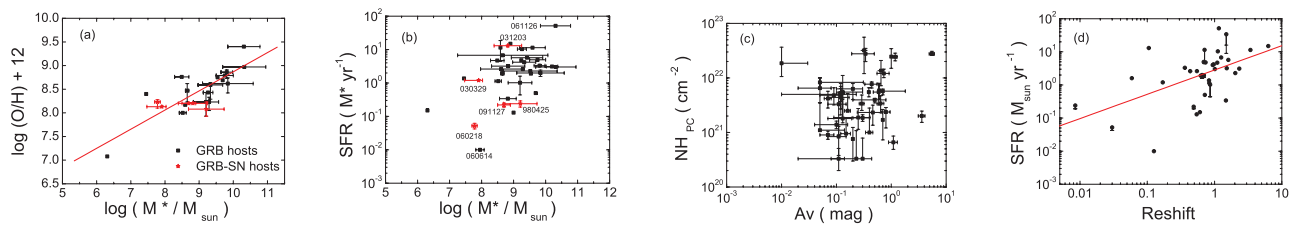

Figure 1. (a) and (b)-Metallicity and star formation rate as a function of Stellar messes; (c) $N_{H}$ value as a function of $A_{V}$ for the GRB host galaxies, where $N_{H}$ and $A_{V}$ are measured from $\mathrm{X}$-ray and optical observations, respectively; (d)Star formation rate as a function of redshift. Lines are the best to the data.

\section{References}

Savaglio, S., Glazebrook, K., \& Le Borgne, D. 2009, ApJ, 691, 182

Kewley, L. J. \& Dopita, M. A. 2002, ApJS 142, 35

Jie-Min Chen, Lan-Wei Jia, \& En-Wei Liang 2012, in preparation

$\dagger$ Supported by the National Natural Science Foundation of China (Grants No. 11025313 and 11203008), the "973" Program of China (2009CB824800), and the Guangxi Natural Science Foundation (2010GXNSFA013112 and Contract No. 2011-135). 